\title{
Frauen machen Karriere!
}

\section{Petra Pflugfelder}

Manager Learning\&Professional Skills Development im Bereich Personal bei der Coca-Cola Erfrischungsgetränke AG, Berlin

Das Thema Frauen und Karriere hat Hochkonjunktur und ist in den Medien präsent wie lange nicht mehr. In vielen großen Unternehmen ist das Thema Diversity auf der strategischen Agenda, meist mit dem Schwerpunkt, mehr Frauen in die Führungsebenen zu bringen. Selbst die Diskussion um eine Frauenquote in Unternehmen wird wieder geführt, vor allem in Hinblick auf Aufsichtsrats- und Vorstandspositionen. Früher waren oft die Frauen selbst die schärfsten Kritikerinnen der Quote. Dies ändert sich: 51 Prozent der Frauen, die vom Institut Sinus Sociovision $2010^{1}$ befragt wurden, sprachen sich für eine Quote für Aufsichtsratsgremien aus. Das Institut hat über 500 Führungskräfte in der deutschen Wirtschaft befragt. Von ihnen bezweifeln zudem 70 Prozent (Frauen sowie Männer), dass das Ziel „mehr Frauen in Führungspositionen“von alleine gelingt.

Die Deutsche Telekom hat sich mit der Veröffentlichung der Zielsetzung, bis 2015 ihre Führungspositionen zu 30 Prozent mit Frauen zu besetzen, an die Spitze einer Bewegung gesetzt, die hoffentlich bald weitere Unternehmen zu ähnlichen Schritten motiviert.

Statistisch gesehen hat sich nicht viel verändert in den vergangenen Jahren (ohne Quote!). Nur 2,5 Prozent der Vorstandsposten in den 200 umsatzstärksten Unternehmen in Deutschland sind weiblich besetzt. Bei den Aufsichtsräten sieht es etwas besser aus: Zehn Prozent Frauenanteil bei den Top 200-Unternehmen. Der größte Teil dieser Frauen wird allerdings von Arbeitnehmerseite nominiert. ${ }^{2}$

Der Anteil von Frauen im Management in der deutschen Wirtschaft beträgt durchschnittlich 19 Prozent. Dabei ist der Anteil in Großkonzernen und DAX-Unternehmen geringer und liegt bei 14 (Top 200) bzw. 9,4 Prozent (Top 100). Mit einem Anteil von 38 Prozent sind Frauen in Kleinunternehmen im mittleren Management am besten vertreten. ${ }^{3}$

Demgegenüber steht die Anzahl qualifizierter Frauen, die in die Unternehmen strömen und ihre Chancen einfordern. Frauen stellen mittlerweile 51 Prozent der Hochschulabgänger ${ }^{4}$ (gleicher Anteil in den Rechtswissenschaften).

Dass es einen wirtschaftlichen Vorsprung bringt, mehr Frauen in Führungsgremien zu haben, zeigen eine wachsende Zahl von Studien auf. Eine der ersten war „Women Matter“ der McKinsey Company ${ }^{5}$, die nachweist, dass Unternehmen mit einem höheren Anteil an Frauen in Entscheidergremien verglichen zum Industriedurchschnitt ein besseres wirtschaftliches Ergebnis erzielen.

Soweit die Welt der Statistiken. Wie ist die gefühlte Realität am Arbeitsplatz? Auf was sollten Frauen - und gerade auch Berufsanfängerinnen - vorbereitet sein, wenn sie in Unternehmen Karriere machen wollen?
Die Luft wird nach oben hin dünner. Das heißt, der Frauenanteil sinkt sukzessive in den mittleren und höheren Führungsebenen.

Um die erste Karrierestufe zu erklimmen, sind vorwiegend fachliche Kompetenz und Leistung, Zuverlässigkeit, Einsatzbereitschaft, Fleiß gefragt. Die beste Fachkraft hat gute Chancen auf die Teamleitung.

Anders sieht es in der nächsten Karrierephase aus. Um in die mittlere, obere Führungsebene zu kommen, sind noch andere Faktoren wirksam.

Sich und seine Leistung offensiv zu vermarkten, dafür zu sorgen, dass man von den Entscheidern wahrgenommen wird, ist einer der Faktoren. Es geht darum zu verstehen, wie die männlichen Kollegen kommunizieren und welchen Spielregeln sie folgen. Auch mal die Ellenbogen auszufahren und in Konkurrenz zu gehen fällt Frauen deutlich schwerer. In der von männlichen Werten und Kommunikationsverhalten geprägten Unternehmenswelt sind Fähigkeiten gefragt, die Frauen in ihrer Sozialisation oft nicht gelernt haben.

Netzwerken ist ein weiteres Thema, womit sich Frauen noch schwertun. Seilschaften bilden, Bündnisse schließen wie die Männer - bei vielen Frauen hat das nach wie vor einen faden Beigeschmack („Vitamin B brauche ich nicht!“). Und sie trennen häufig bewusst zwischen Arbeitswelt und Privatleben, bevorzugen das Netzwerken im familiären Umfeld und im Freundeskreis. Förderlich für die Karriere ist aber, sich innerhalb und außerhalb des Unternehmens aktiv zu vernetzen. In reinen Frauennetzwerken ${ }^{6}$, aber auch in gemischt-geschlechtlichen. Denn Karriere funktioniert - nicht nur, aber auch über das gute alte Prinzip des Gebens und Nehmens.

Wie viele große Unternehmen bietet auch die Coca-Cola Erfrischungsgetränke AG spezielle Entwicklungsmaßnahmen für Frauen an. Eine unserer erfolgreichsten Maßnahmen ist das Mentoring: die Beratung und Begleitung durch einen erfahrenen Mentor. Mentoringprogramme entfalten ihre Kraft durch die Vorbildfunktion, die ein Mentor einnimmt. Noch sind weibliche Vorbilder in Top-Positionen eher rar, aber wichtig. Auf ihrem Karriereweg hatten fast alle Führungskräfte einen oder mehrere informelle Förderer. Nebenbei entstehen im Mentoring Netzwerke, die noch lange wirken.

1 Studie der Sinus Sociovision $\mathrm{GmbH}$ 2010, Frauen in Führungspositionen - Barrieren und Brücken.

2 Elke Holst: „Frauen in Spitzengremien großer Unternehmen weiterhin massiv unterrepräsentiert", Wochenbericht des DIW Berlin Nr. 4/2010.

3 Hoppenstedt Firmeninformation $\mathrm{GmbH}$, Stand Mai 2009; Definitionen: Großunternehmen: 20 Mio. Euro Umsatz und mehr, mehr als 200 MA; Mittelständische Unternehmen: 2-20 Mio. Euro Umsatz und 20-200 MA; Kleinunternehmen: Bis 2 Mio. Euro Umsatz und weniger als $20 \mathrm{MA}$.

4 Studie der Sinus Sociovision $\mathrm{GmbH}$ 2010, Frauen in Führungspositionen - Barrieren und Brücken.

5 McKinseyCompany, 2007, A wake-up call for female leadership. 
Manche scheuen davor zurück, spezielle Angebote wie Förderprogramme, Seminare, Netzwerke und ähnliches für Frauen zu nutzen. Sie befürchten, dass ihnen dies als Schwäche ausgelegt wird. Sicher haben viele Frauen auch ohne diese „Hilfsmittel“ Karriere gemacht. Aber warum nicht gut vorbereitet den Wettbewerb antreten? Die Wahrnehmung zu schärfen für die Mechanismen, die wirken?

Ein Anfang kann auch einer der vielen Ratgeber sein, die momentan auf dem Markt sind. Sie vermitteln einen Einstieg ins Thema und geben gute Tipps. Als ein Beispiel sei „Spiele mit der Macht - wie Frauen sich durchsetzen“ von Marion Knaths genannt: „Verstehen Sie sich als Spielerin in einem Spiel, dessen Regeln sich wie eine Fremdsprache erlernen lassen."

6 Beispiele für Frauennetzwerke sind: www.ewmd.org, www.bpwgermany.de, www.europeanpwn.net (Zugriff jeweils 14.7.2010).

\section{Wie ich wurde, was ich (auch) bin}

\section{Mechthild Rawert (SPD), MdB}

Einzelne Lebensfäden der eigenen 52-jährigen Biografie zu betrachten, bedeutet den Blick zurück nach vorne richten. Spannend, wo gesellschaftlicher Wandel, wo Stagnation.

Feministinnen meines Alters sind geprägt durch das Prinzip der Zweiten Frauenbewegung „Das Private ist politisch“, durch parteiisches Agieren gegen patriarchale Gesellschaftsstrukturen. Schlüsselthemen waren „Arbeit“, „Sexualität“ und „Beziehungen“, war das Streben nach Autonomie. Noch heute existieren Geschlechterhierarchien zum Nachteil von Frauen, ist „Arbeit und Geschlecht“ eine politische Herausforderung auch in Bezug auf unbezahlte Hausarbeit, unbezahlte und bezahlte Versorgungsarbeit („care“), zivilgesellschaftliches Engagement. „Wer die menschliche Gesellschaft will, muss die männliche überwinden “ - so die Herausforderung im sozialdemokratischen „Hamburger Programm“. Parteiisch und parteilich zu sein bedeutet Politik gegen jede Form von struktureller und individueller Diskriminierung, bedeutet Einsatz für soziale Demokratie und Teilhaberechte, für Chancengleichheit und sozialen Aufstieg, für gelebte Vielfalt in einer Einwanderungsgesellschaft.

\section{Bildungsstart und Bildungsübergänge als Chance oder Barriere}

Aufgewachsen als Älteste von sieben Kindern in einem katholischen, parteipolitisch konservativen bäuerlichen Elternhaus gehörte ich soziologisch zur Gruppe der im Bildungssystem der 60er Jahre besonders benachteiligten „katholischen Mädchen vom Lande“. Dass dieses für mich nicht zur Lebensrealität wurde, verdanke ich vor allem meiner Mutter, die - obwohl selbst kriegsbedingt am Studium gehindert - allen Kindern eine erfolgreiche Bildungsmanagerin war. So erhielten auch die vier Mädchen den „Auftrag“, die bestmögliche Berufsausbildung zu absolvieren, selbständig zu sein. Ich besuchte das Mädchengymnasium in Coesfeld/ NRW, machte 1976 Abitur. Aussteuer und Hochzeitstruhe spielten keine Rolle. Eine eigene Familie gründen, Familie als generationenübergreifende Gemeinschaft achten allerdings schon. Sozialisatorische Beigaben waren auch ein hohes Ar- beitsethos sowie die Bereitschaft zu zivilgesellschaftlichem Engagement.

Ich bin davon überzeugt, dass die Befähigung der Eltern, den Bildungsweg ihrer Kinder mitzugestalten, sie auf eine offene Gesellschaft vorzubereiten, Voraussetzung einer gelingenden Bildungsförderung und Integration ist.

\section{Frauen auf dem Sprung}

Der Übergang Schule-Ausbildung bzw. Schule-Studium ist nach wie vor geschlechtsspezifisch geprägt. Seit Längerem findet eine stille Revolution statt: Junge Frauen sind davon überzeugt: „Ich bin gut in dem, was ich mache“, haben hohe Ansprüche an ihre beruflichen Perspektiven, wollen auf eigenen Beinen stehen und Karriere machen (vgl. Brigitte-Studie). Dieses auf ihrem Bildungsvorsprung beruhende neue Selbstbewusstsein der jungen Frauen gilt es politisch und gesellschaftlich zu unterstützen. Schließlich muss sich die „Bildungsrendite" (Jutta Allmendinger) junger Frauen verbessern, denn noch entscheiden sich trotz besserer Schulabschlüsse junge Frauen überproportional häufig gegen ein Studium, zumindest gegen ein sogenanntes MINT-Studium. Geschlechtsspezifisch wirkende Barrieren sind aktuell auch die Studiengebühren, die schwarz-gelbe Elite-Förderung statt allgemeiner BAföG-Erhöhungen.

Ich studierte Sozialpädagogik an der Katholischen Fachhochschule Münster und war während dieser Zeit in der studentischen Selbstverwaltung sowie auf Bundesebene in der Jugendverbandsarbeit aktiv. Mein persönlicher Sprung war Berlin.

\section{Berlin, mon amour}

Seit fast 30 Jahren lebe ich in Berlin, habe mich mehrmals in dieser Zeit für West-Berlin bzw. Berlin als Lebensort entschieden. Berlin fordert mich ständig heraus, ist als Metropole und Kiez häufig gesamtgesellschaftlicher Experimentierraum, steht für Emanzipation und politischen Aufbruch - so zum Beispiel am 17. März 1989 mit dem ersten rot-grünen Berliner „Frauensenat". Acht Senatorinnen bewiesen unter den Argusaugen der Medien, dass „Frauen und Macht“ nicht zum Zusammenbruch der Gesellschaft führt. Männer müssen lernen, die 